\title{
Nano-structural analysis by cryo-TEM of aqueous solutions of a hybrid anionic surfactant: relation between structure and rheological properties
}

\author{
Dganit Danino
}

Department of Food Engineering and Biotechnology, Technion-Israel Institute of Technology, Haifa 32000, Israel.

Self-assembly is a general, fundamental phenomenon, where physical interactions control the spontaneous association of molecules into nano-sized organized, dynamic, and thermodynamically stable structures. The shape of these structures depends on the chemical structure as well as on external forces such as temperature, concentration, salinity and $\mathrm{pH}$. Direct relationship exists between the shape of structures and dynamic properties like the critical micelle concentration, surface tension and viscosity.

The anionic hybrid fluorocarbon/hydrocarbon surfactant sodium 1-oxo-1[4(tridecafluorohexyl)phenyl]-2-hexanesulfonate (FC6HC4) displays a peculiar rheological behavior. At $25{ }^{\circ} \mathrm{C}$ the viscosity of $\mathrm{FC} 6 \mathrm{HC} 4$ solutions goes through a maximum at a surfactant concentration of about $9 \mathrm{wt} \%$, then through a minimum at about $16 \mathrm{wt} \%$. The effect of temperature on the viscosity of the $10 \mathrm{wt} \%$ FC6HC4 solution is even more striking. The viscosity increases with temperature up to a maximum value at $36{ }^{\circ} \mathrm{C}$ (Tobita et al., Langmuir 1997, 13, 5054), then decreases rapidly at higher temperatures. The system is viscoelastic in the temperature range around the viscosity maximum, a behavior referred to as thermoresponsive viscoelasticity. This rheological behavior noted for FC6HC4 solutions is of interest for applications for which fluids that become more viscous at high temperature are required. For instance the oil industry requires fluids of very high viscosity at high temperature for cracking oil-containing rock. Injecting such fluids into the oil well is difficult because of their high viscosity at ambient temperature. A fluid like FC6HC4 that displays low viscosity at low temperature and becomes highly viscous at the elevated temperature of the oil-containing rock would greatly facilitate the process.

To explain the behavior of FC6HC4 we have correlated the observed rheological behavior with the nanostructural analysis of the assemblies formed in aqueous solutions, using digital-imaging transmission electron microscopy at cryogenic temperatures (digitalimaging cryo-TEM) that we have developed and advanced in recent years [1]. Cryo-TEM is based on the ultrafast vitrification of a thin liquid film of the investigated solution that ensures minimal, if any, structural changes of the assemblies present in the solution. The use of a CCD camera allows detailed and reliable characterizations at low-dose exposures to avoid radiation damage, which makes the method indispensable for the study of a wide variety of soft materials and complex fluids. Our detailed study of FC6HC4 solutions as a function of concentration and temperature permit us to explain the effect of concentration on the solution viscosity and the thermoresponsive behavior [2]. A few of the structures found as a function of temperature and concentration are presented in the images below. Especially interesting is the multiconnected disordered phase, which is possibly the L3 phase, seen for the first time by cryo-TEM.

[1] Danino et al, Colloids and Surfaces A: Physicochemical and Engineering aspects 183, 113 (2001).

[2] Danino et al, J. Colloid Interface Science (accepted). 

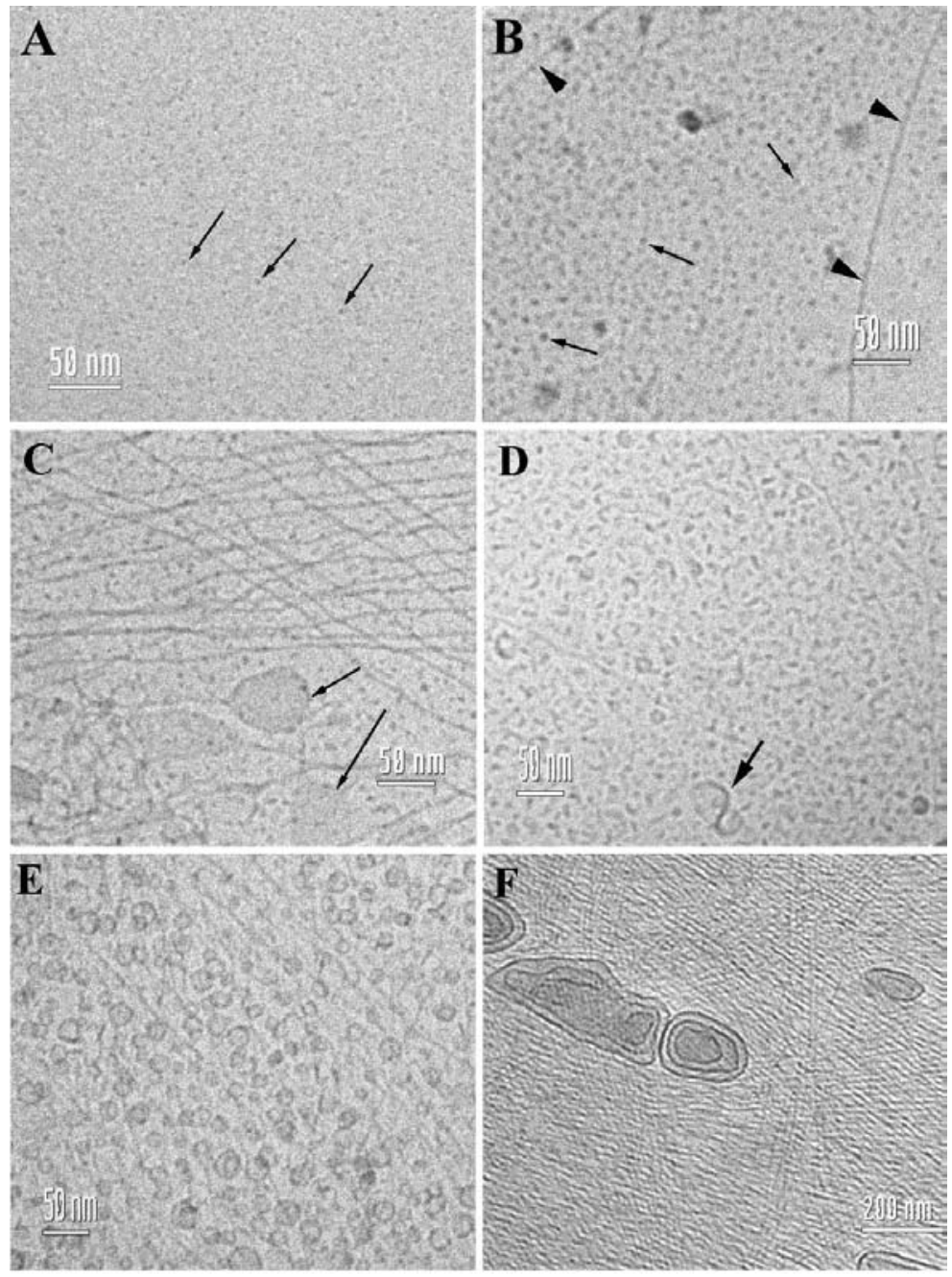

Electron micrographs of vitrified FC6HC4 solutions of increasing concentration at $20{ }^{\circ} \mathrm{C}$ : (A) $0.5 \mathrm{wt} \%$ solution showing only very small spheroidal micelles (arrows); at $2 \mathrm{wt} \%$; spheroidal micelles (arrows) coexist with rod-like micelles (arrowheads) in (B), and some membrane patches (arrows) in (C); (D) at $3.5 \mathrm{wt} \%$ small disk-like micelles and vesicles are the dominant features; note twisted disk-like micelle (arrow); (E) $5 \mathrm{wt} \%$ solution shows many rod-like micelles coexisting with a large number of mostly small vesicles; (F) $10 \mathrm{wt} \%$ - many rod-like micelles coexisting with few distorted multilamellar vesicles.
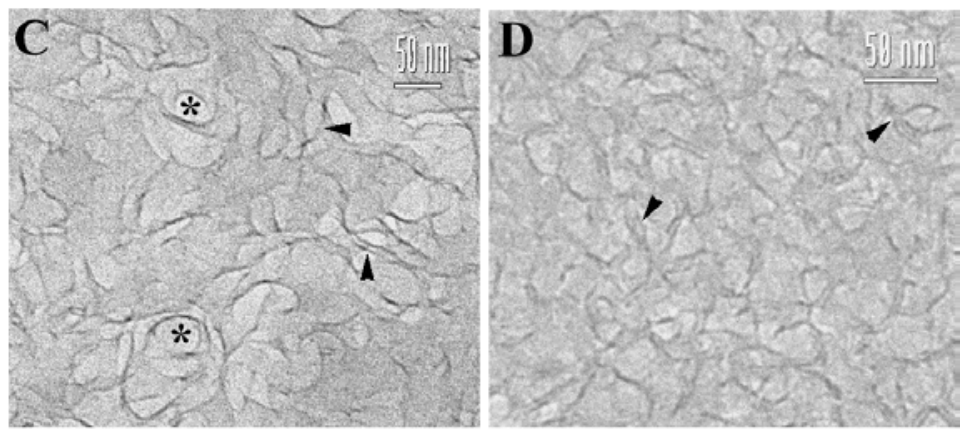

Electron micrographs of vitrified specimens of $10 \mathrm{wt} \%$ FC6HC4 solutions quenched from (C) $50{ }^{\circ} \mathrm{C}$ and (D) $60{ }^{\circ} \mathrm{C}$. Both show a disordered lamellar possibly $\mathrm{L}_{3}$ phase, seen by cryoTEM for the first time. Arrowheads point to membrane edges and asteriks indicate superposition of holes over membranes. 